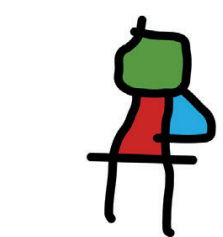

SEICAIP

\section{Allergologia et immunopathologia}

Sociedad Española de Inmunología Clínica, Alergología y Asma Pediátrica

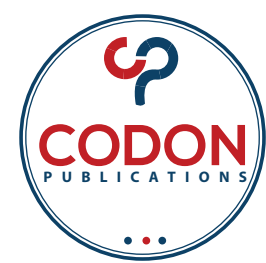

RESEARCH ARTICLE

OPEN ACCESS (C) (1) (2)

\title{
The IncRNA PVT1/miR-423-5p axis is positively correlated with the severity of bronchial asthma
}

\author{
Ju Qiu ${ }^{a}$ Kai Zhang ${ }^{b}$, Jing Liuc, Dianfeng Cao ${ }^{a *}$, Ping Gao ${ }^{a}$, Baoling Zhang ${ }^{a}$, Na Zhao ${ }^{a}$, \\ Ruofang Wang ${ }^{a}$, Xinsheng Shi ${ }^{a}$
}

\begin{abstract}
${ }^{a}$ Department of Occupational Diseases, Zibo Hospital for the Prevention and Treatment of Occupational Diseases, Zibo, China ${ }^{b}$ Department of Burn, Zibo Hospital for the Prevention and Treatment of Occupational Diseases, Zibo, China

'Department of Respiratory Medicine, Zibo Central Hospital, Zibo, China
\end{abstract}

Received 24 June 2021; Accepted 9 October 2021

Available online 2 January 2022

\section{KEYWORDS \\ bronchial asthma; \\ LnCRNA PVT1; \\ miR-423-5p; \\ receiver operating characteristic curve; serum}

\begin{abstract}
Bronchial asthma (BA) is a serious problem affecting the quality of life of patients. Long noncoding RNAs (IncRNAs) are involved in BA. This study set out to investigate expressions of PVT1/miR-423-5p in the serum of BA patients and its clinical value on BA diagnosis and evaluation. This study included the same number $(N=100)$ of patients with $B A$ at remission (BA-R), $B A$ at exacerbation (BA-E), and healthy controls. PVT1 level was increased in BA-R and BA-E patients, and PVT1 level was higher in BA-E patients than BA-R patients. miR-141-3p targeted PVT1. miR-423-5p was downregulated in the serum of BA patients and was negatively correlated with PVT1. Area under ROC curve of PVT1/miR-423-5p axis on BA-R patients was 0.837 with sensitivity 0.74 , specificity 0.84 , while that of BA-E was 0.974 with sensitivity 0.87 and specificity 0.95 . PVT1/miR-423-5p axis was negatively correlated with $\mathrm{FEV}_{1} / \mathrm{FVC}, \mathrm{FEV}_{1} \%$ pred, and IL-10, and positively correlated with IgE, TNF-a, and IL-6. PVT1 and PVT1/miR-423-5p axis were associated with increased severity while miR-423-5p axis was negatively associated with BA severity. In conclusion, increased levels of PVT1/miR-423-5p had higher diagnostic efficiency on BA patients, especially patients with acute exacerbation.

(c) 2022 Codon Publications. Published by Codon Publications.
\end{abstract}

*Corresponding author: Dianfeng Cao, Department of Occupational Diseases, Zibo Hospital for the Prevention and Treatment of Occupational Diseases, Zibo Sixth People’s Hospital, 121 Nanjing Road, Zhangdian District, Zibo City, 255000, Shandong Province, Zibo, China. Email address: dianfeng0309@163.com 


\section{Introduction}

Bronchial asthma (BA) is a prevalent chronic disease of the airway which involves many cells and cellular components. ${ }^{1}$ Asthma is a complex syndrome with a variety of pathophysiological mechanisms. ${ }^{2}$ Recent data showed that BA has affected more than $10 \%$ of the population and the prevalence continues to increase. ${ }^{3} \mathrm{BA}$ at remission (BA-R) is the absence of asthma symptoms for a period of time, ${ }^{4}$ while $B A$ at exacerbation (BA-E) is a progressive increase in asthma symptoms. ${ }^{5}$ In recent years, the incidence and mortality rate of $\mathrm{BA}$ is on the rise due to the deterioration of environmental pollution, which adversely impacts the health condition and the quality of life of BA patients. ${ }^{6,7}$ Therefore, seeking novel biomarkers for BA diagnosis and treatment is critical for BA patients.

Long noncoding RNAs (IncRNAs) are a heterogeneous group of noncoding RNAs with a length of 200-100,000 nt. ${ }^{8}$ LncRNAs regulate protein expression or transcriptional and post-transcriptional level, and participate in the development of multiple diseases including asthma through many mechanisms. ${ }^{9,10}$ Based on the existing study, IncRNA plasmacytoma variant translocation 1 (PVT1) might be important in asthma and IncRNA PVT1 exacerbates inflammation and cell-barrier injury during asthma by modulating miR149. ${ }^{11}$ According to the study of Austin et al., PTV1 was upregulated in the primary airway smooth muscle cells of patients with asthma and PVT1 expression might affect the proliferation of airway smooth muscle cells and the release of interleukin (IL)-6.12 However, the expression of IncRNA PVT1 in the serum of BA patients and whether IncRNA PTV1 could be used for BA diagnosis and treatment require further investigation.

The biological function of IncRNA PTV1 needs to be clarified. Accumulating evidence has reported the effect of IncRNA/microRNAs (miRNAs) in BA, indicating its association with the etiology of BA..$^{13-15}$ The downstream miRNAs of InCRNA PVT1 were obtained through bioinformatics analysis. miR-423-5p, as one of the downstream miRNAs, was downregulated in the Th2-pretreated human airway epithelial cells, which suggested that miR-423-5p might be critical in changing the pro-inflammatory and anti-viral responses in BA-E patients. ${ }^{16}$ But, the expression of miR-423-5p in the serum of BA patients, and the association of the IncRNA PVT1/miR-423-5p axis with the risk, severity, and inflammatory cytokines of BA has not been reported at home and abroad. This study intended to study the expressions of IncRNA PVT1/miR-423-5p and explore the clinical values of the IncRNA PVT1/miR-423-5p axis on BA diagnosis and severity evaluation.

\section{Material and Methods}

\section{Ethics statement}

This study was authorized by the Ethics Committee of Zibo hospital for the prevention and treatment of occupational diseases. All participants signed the informed consent before being recruited for the study.

\section{Participants}

In this study, 100 patients with BA-E and 100 patients with $B A-R$ were recruited in Zibo hospital for the prevention and treatment of occupational diseases between June 2019 and June 2020. The diagnosis accorded with the Global Initiative for Asthma (GINA) guidelines (2016). The inclusion criteria for BA-E patients were: (1) diagnosed as BA according to the guideline; (2) manifested with exacerbated symptoms including dyspnea, chest tightness, and serious cough; and (3) aged above 18 years. The inclusion criteria for BA-R patients were: (1) diagnosed as BA according to the guideline; (2) with clinical remission status, the symptoms disappeared and pulmonary function was improved to the pre-acute level and maintained at least for 3 months; and (3) aged above 18 years. The exclusion criteria were: (1) cardiac asthma, endometrial lesions of trachea, bronchogenic carcinoma, or allergic pulmonary infiltration; (2) hematological diseases, autoimmune disorders, or serious infections; (3) solid tumors or malignancies; and (4) pregnant or lactating women. And, 100 healthy controls in the physical examination center of Zibo hospital for the prevention and treatment of occupational diseases during the same period were included in this study. The healthy controls had normal lung function, and no history of asthma, autoimmune diseases, allergic diseases, blood diseases, severe infection, or malignant tumors.

\section{Data and sample collection}

Characteristics of all participants, including age, gender, and family history, were recorded. The laboratory examinations such as immune globulin E (IgE) and pulmonary ventilation function including forced expiratory volume in $1 \mathrm{~s}\left(\mathrm{FEV}_{1}\right)$ and forced vital capacity (FVC) were recorded, followed by the calculation of $\mathrm{FEV}_{1} / \mathrm{FVC}$ and $\mathrm{FEV} \%$ predicted $\left(\mathrm{FEV}_{1} \%\right.$ pred). The exacerbation severity of BA-E patients were assessed based on the Global Strategy for Asthma Management and Prevention (updated 2010) (www. ginasthma.org) on the day of hospital admission. Moreover, blood samples were collected using ethylenediaminetetraacetic acid tubes after confirming eligibility, followed by centrifugation at $1000 \mathrm{~g}$ for $15 \mathrm{~min}$. The plasma was isolated and stored at $-80^{\circ} \mathrm{C}$ for further examination.

\section{Enzyme-linked immunosorbent assay (ELISA)}

Levels of pro-inflammatory cytokines tumor necrosis factor- $\alpha$ (TNF- $\alpha$ ), IL-6, and anti-inflammatory cytokine IL-10 in the serum were determined using ELISA kits (R\&D Systems, Minneapolis, MN, USA) as per the instructions. The sample was incubated with coating solution at $37^{\circ} \mathrm{C}$ for $2 \mathrm{~h}$ in ELISA plates at $4^{\circ} \mathrm{C}$ overnight. Then, the sample was sealed with $10 \%$ bovine serum albumin (BSA) at $4^{\circ} \mathrm{C}$ overnight. After rinsing, the sample was incubated with primary antibodies at $37^{\circ} \mathrm{C}$ for $2 \mathrm{~h}$ and with secondary antibodies for $1 \mathrm{~h}$. Termination solution was added after color reaction. Absorbance of each well at $450 \mathrm{~nm}$ was detected. The detailed information of ELISA kits were as follows: TNF-a (SRTA00, Range: 12.5-800 pg/mL, Sensitivity: 5 pg/mL), IL-6 (SR6000B, Range: 
62.5-4000 pg/mL, Sensitivity: 36 pg/mL), and IL-10 (SR1000, Range: $31.2-2000 \mathrm{pg} / \mathrm{mL}$, Sensitivity: $10 \mathrm{pg} / \mathrm{mL}$ ).

\section{Reverse transcription-quantitative polymerase chain reaction ( $R T-q P C R)$}

The total RNA was extracted using TRIzol reagent (Invitrogen, Carlsbad, CA, USA) and transcribed into CDNA using PrimeScript RT reagent kit (Takara, Dalian, China). qPCR was conducted on ABI7900HT fast PCR real-time system (Applied Biosystems, Foster city, CA, USA) using SYBR® Premix Ex $\mathrm{Taq}^{\mathrm{TM}}$ II (Takara). Reaction conditions were as follows: predenaturation at $95^{\circ} \mathrm{C}$ for $10 \mathrm{~min}$ and 40 cycles of denaturation at $95^{\circ} \mathrm{C}$ for $10 \mathrm{~s}$, annealing at $60^{\circ} \mathrm{C}$ for $20 \mathrm{~s}$ and extension at $72^{\circ} \mathrm{C}$ for $34 \mathrm{~s}$. Data were analyzed using the $2^{-\Delta \Delta \mathrm{ct}}$ method, with GAPDH and U6 as internal controls. ${ }^{17}$ Primers were synthesized by Shanghai Sangon Biotech Co., Ltd. (Shanghai, China). Primer sequences are illustrated in Table 1.

\section{Bioinformatics analysis}

The downstream target miRNAs of IncRNA PVT1 were predicted through ENCORI (http://starbase.sysu.edu.cn/agoClipRNA.php?source=IncRNA) and LncBase database (http:// carolina.imis.athena-innovation.gr/diana_tools/web/index. php? $r=$ Incbasev2\%2Findex-predicted). Intersections were taken and screened to obtain the target miRNA.

\section{Dual-luciferase reporter assay}

The binding sites of IncRNA PVT1 and miR-423-5p were predicted through Starbase (http://starbase.sysu.edu. cn/agoClipRNA.php?source=lncRNA). The complementary sequences and mutant sequences of miR-423-5p and PVT1 were amplified and cloned onto pmiRGLO dual-luciferase reporter gene vectors (Promega, Madison, WI, USA). The wild-type plasmid PVT1-WT and mutant plasmid PVT1-MUT were constructed and transfected with mimic NC or miR423-5p mimic (GenePharma, Shanghai, China) into HEK293T cells (Shanghai Institute of Biochemistry and Cell Biology, Chinese Academy of Sciences, Shanghai, China) using Lipofectamine $^{T M} 2000$ (Invitrogen) as per the instructions. Luciferase activity was detected after $48 \mathrm{~h}$ of transfection.

\section{Statistical analysis}

SPSS 21.0 (IBM Corp., Armonk, NY, USA), GraphPad Prism 8 (GraphPad Software Inc., San Diego, CA, USA), and Medcalc $®$ version 15.0 (Medcalc Software Ltd, Ostend, Belgium) statistical softwares were adopted for data analysis and graphing. Shapiro-Wilk test (W-test) was used to identify the normal distribution of data. Variable data were described as mean \pm standard deviation or counts. Comparison between two groups was analyzed using the unpaired t-test or $\mathrm{x}^{2}$ test, while comparison among multiple groups was analyzed using one-way analysis of variance (ANOVA) and Tukey's multiple comparisons test. The diagnostic value of IncRNA PVT1/miR-423-5p on BA was analyzed using receiver operating characteristic (ROC) curve. The differences of areas under the ROC curves were analyzed using MedCalc-Comparison of ROC curves. $\mathrm{P}<0.05$ was statistically significant.

\section{Results}

\section{Comparative analysis of clinical data of $B A$ patients and healthy controls}

This study included 100 patients with BA-R, 100 patients with $B A-E$, and 100 healthy controls. The severity of BA-E patients was assessed and BA-E patients were further assigned into mild (31 cases), moderate (43 cases), and severe BA-E patients ( 26 cases). The clinical parameters of BA patients and healthy controls were compared and analyzed, and the result showed no significant difference in clinical baseline parameters (age, gender, and family history) and the data were comparable. The results of blood biochemical parameters showed that the IgE level was significantly increased in BA-R and BA-E patients, and the IgE level of BA-E patients was higher than that of $B A-R$ patients. The examination of lung function parameters showed that $\mathrm{FEV}_{1} / \mathrm{FVC}$ and $\mathrm{FEV}_{1} \%$ pred were significantly reduced in $B A-R$ and $B A-E$ patients, and the values of $B A-E$ patients was lower than that of BA-R patients. The inflammatory cytokine level showed that levels of TNF- $a$ and IL- 6 were increased while IL-10 level was decreased in $B A-R$ and BA-E patients, and the differences between BA-R patients and BA-E patients were significant (all $P<0.001$, Table 2).

Table 1 Primer sequences.

\begin{tabular}{lcc}
\hline Gene & Forward 5'-3' & Reverse 5'-3' \\
\hline LnCRNA PVT1 & TGAGAACTGTCCTTACGTGACC & AGAGCACCAAGACTGGCTCT \\
GAPDH & CTCAGACACCATGGGGAAGGTGA & ATGATCTTGAGGCTGTTGTCATA \\
miR-423-5p & GCCCCCAGCGCCCAAC & AGTGCAGGGTCCGAGGTATT \\
U6 & ATTGGAACGATACAGAGAAGATT & GGAACGCTTCACGAATTTC \\
\hline
\end{tabular}

Note: IncRNA, long noncoding RNA; PVT1, plasmacytoma variant translocation 1; GAPDH, glyceraldehyde-3-phosphate dehydrogenase; miR, microRNA. 
Table 2 Comparative analysis of clinical data of BA patients and healthy controls.

\begin{tabular}{|c|c|c|c|c|c|c|c|}
\hline $\begin{array}{l}\text { Parameters } \\
(\mathrm{N}=100)\end{array}$ & & $\begin{array}{l}\text { (Control) } \\
(\mathrm{N}=100)\end{array}$ & $\begin{array}{c}(B A-R) \\
(N=100)\end{array}$ & $(B A-E)$ & $\mathrm{P}_{\mathrm{a}}$ & $\mathrm{P}_{\mathrm{b}}$ & $P_{c}$ \\
\hline Age (years) & & $38.85 \pm 5.45$ & $39.36 \pm 5.83$ & $40.04 \pm 5.92$ & 0.524 & 0.141 & 0.414 \\
\hline \multirow[t]{2}{*}{ Gender. } & Male & 56 & 61 & 59 & 0.566 & 0.775 & 0.885 \\
\hline & Female & 44 & 39 & 41 & & & \\
\hline \multirow[t]{2}{*}{ Family history of asthma } & No & 86 & 78 & 75 & 0.197 & 0.073 & 0.739 \\
\hline & Yes & 14 & 22 & 25 & & & \\
\hline IgE (IU/mL) & & $32.53 \pm 15.42$ & $96.17 \pm 15.29$ & $249.25 \pm 30.36$ & $<0.001$ & $<0.001$ & $<0.001$ \\
\hline $\mathrm{FEV}_{1} / \mathrm{FVC}(\%)$ & & $87.49 \pm 6.25$ & $80.02 \pm 6.66$ & $60.13 \pm 6.51$ & $<0.001$ & $<0.001$ & $<0.001$ \\
\hline FEV $\%$ pred (\%) & & $98.08 \pm 5.03$ & $83.15 \pm 5.05$ & $66.73 \pm 5.21$ & $<0.001$ & $<0.001$ & $<0.001$ \\
\hline TNF-a (pg/mL) & & $15.45 \pm 5.67$ & $26.60 \pm 9.33$ & $68.19 \pm 8.35$ & $<0.001$ & $<0.001$ & $<0.001$ \\
\hline IL-6 (pg/mL) & & $8.59 \pm 5.15$ & $19.91 \pm 7.63$ & $43.58 \pm 8.77$ & $<0.001$ & $<0.001$ & $<0.001$ \\
\hline IL-10 (pg/mL) & & $21.09 \pm 5.26$ & $15.33 \pm 5.85$ & $7.65 \pm 4.01$ & $<0.001$ & $<0.001$ & $<0.001$ \\
\hline \multirow[t]{3}{*}{ Exacerbation severity } & Mild & - & - & 31 & - & - & - \\
\hline & Moderate & - & - & 43 & - & - & - \\
\hline & Severe & - & - & 26 & - & - & - \\
\hline
\end{tabular}

Note: BA-R, bronchial asthma at remission; BA-E, bronchial asthma at exacerbation; $\mathrm{FEV}_{1}$, forced expiratory volume in $1 \mathrm{~s}$; FVC, forced vital capacity; $P_{a} B A-R$ vs Control; $P_{b} B A-E$ vs Control; $P_{c} B A-E$ vs BA-R.

\section{LnCRNA PVT1 was highly expressed in the serum of BA patients}

RT-qPCR showed that the expression of IncRNA PVT1 was significantly increased in the serum of $B A-R$ and $B A-E$ patients, and the PVT1 level of BA-E patients was higher than that of BA-R patients (Figure 1).

\section{miR-423-5p was poorly expressed in the serum of BA patients and negatively correlated with PVT1 expression}

One of the mechanisms of IncRNA in disease development could be explained by competitive endogenous RNA (ceRNA) theory. ${ }^{18}$ The effect of IncRNA/miRNA axis in BA has been reported previously, suggesting that it might be involved in the etiology of BA. ${ }^{13-15}$ To investigate the effect of IncRNA PVT1/miRNA axis in BA, 17 downstream miRNAs were obtained by screening through ENCORI and LncBase databases (Figure 2A). Among the 17 miRNAs, miR-423-5p has been reported to be downregulated in Th2-pretreated human airway epithelial cells, indicating a critical role in changing pro-inflammatory and anti-viral responses during BA-E. ${ }^{16}$ Meanwhile, the target relation of miR-141-3p and PVT1 was verified by dual-luciferase reporter assay (Figure 2B). RT-qPCR showed that miR-423-5p expression was significantly decreased in the serum of $B A-R$ and BA-E patients, and miR-423-5p expression in the serum of BA-E patients was lower than that of BA-R patients (all $P<$ 0.01 , Figure 2 C). In addition, Pearson's correlation analysis showed that expression of IncRNA PVT1 was negatively correlated with miR-423-5p expression ( $P<0.01$, Figure $2 D)$. Briefly, the IncRNA PVT1/miR-423-5p axis might be involved in of BA.

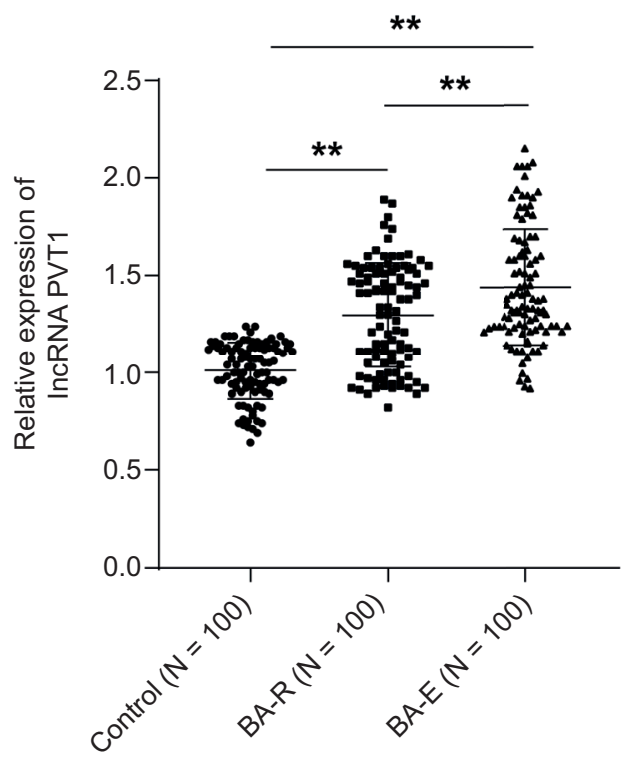

Figure 1 LncRNA PVT1 was highly expressed in the serum of BA patients. LncRNA PVT1 expression in the serum of BA patients and controls was detected by RT-qPCR. Data were expressed as mean \pm standard deviation. Comparison among multiple groups was analyzed using one-way ANOVA, followed by Tukey's multiple comparisons test. ${ }^{* *} \mathrm{P}<0.01$.

\section{LncRNA PVT1/miR-423-5p axis had higher diagnostic efficiency on BA patients}

To further explore the value of IncRNA PVT1/miR-423-5p axis in BA diagnosis, the changes of IncRNA PVT1/miR423-5p expressions in BA patients were analyzed. The 
(A)

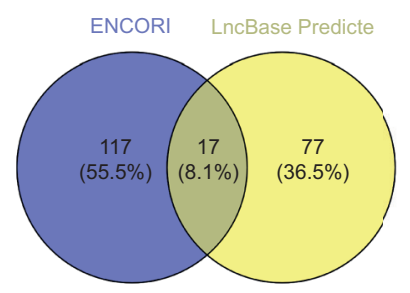

(C)

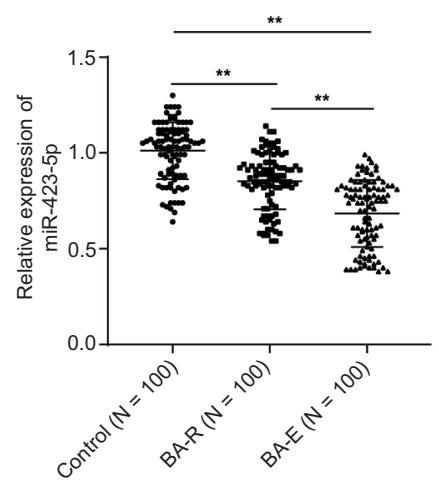

(B)

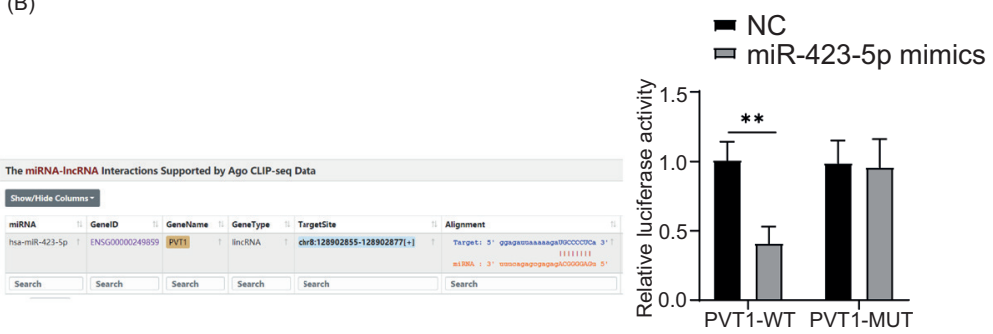

(D)
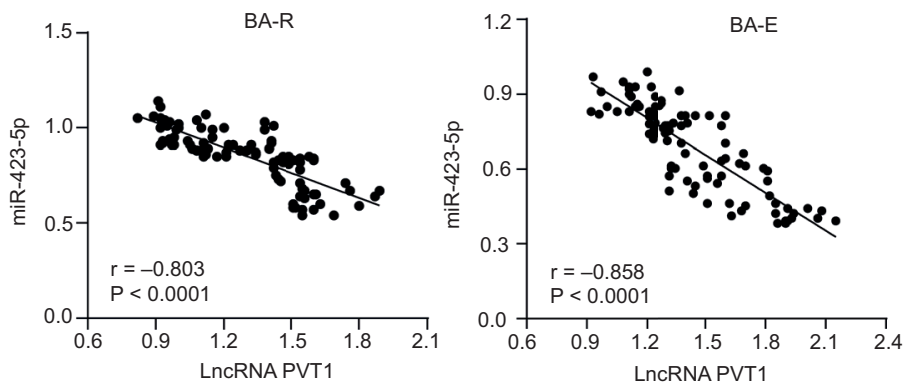

Figure 2 miR-423-5p was poorly expressed in the serum of BA patients and negatively correlated with PVT1 expression. (A) Target miRNAs of IncRNA PVT1 were screened through ENCORI and LncBase databases and intersections were taken; (B) Target relation of miR-141-3p and PVT1 was verified by dual-luciferase reporter assay; (C) miR-423-5p expression was detected by RT-qPCR; (D) Correlation of IncRNA PVT1 and miR-423-5p was analyzed using Pearson's correlation analysis. Data in panel B were analyzed using unpaired $t$-test, while data in panel C were analyzed using one-way ANOVA, followed by Tukey's multiple comparisons test. **P $<0.01$.

result showed that expressions of IncRNA PVT1/miR-423-5p were increased in the serum of BA-R and BA-E patients, and expressions of IncRNA PVT1/miR-423-5p in BA-E patients were higher than that in BA-R patients (all $P<0.01$, Figure $3 A$ ). The diagnostic efficiency of IncRNA PVT1/miR423-5p in BA patients was analyzed through ROC curve. The area under the ROC curve of IncRNA PVT1 diagnosing BA-R patients was 0.784 with sensitivity 0.60 and specificity 0.97; the area under the ROC curve of miR-423-5p diagnosing BA-R patients was 0.775 with sensitivity 0.78 and specificity 0.71 ; the area under the ROC curve of IncRNA PVT1/miR-423-5p diagnosing BA-R patients was 0.837 with sensitivity 0.74 and specificity 0.84 . MedCalc-Comparison of ROC curves analysis showed that the areas under ROC curves among the three presented no significant difference (Figure 3B). In addition, the area under the ROC curve of IncRNA PVT1 diagnosing BA-E patients was 0.930 with sensitivity 0.84 and specificity 0.97 ; the area under the ROC curve of miR-423-5p diagnosing BA-E patients was 0.916 with sensitivity 0.98 and specificity 0.71 ; the area under the ROC curve of IncRNA PVT1/miR-423-5p diagnosing BA-E patients was 0.974 with sensitivity 0.87 and specificity 0.95. MedCalc-Comparison of ROC curves analysis showed that the area under the ROC curve of IncRNA PVT1/miR423-5p axis diagnosing BA-R patients was higher than that of IncRNA PVT1 and miR-423-5p (all $P<0.05$, Figure 3C). The above results indicated that the IncRNA PVT1/miR423-5p axis had higher diagnostic efficiency in BA patients, especially in patients with BA-E.

\section{Correlation analysis of IncRNA PVT1/miR-423-5p axis and clinical parameters}

The correlation of IncRNA PVT1/miR-423-5p axis with biochemical parameters, pulmonary ventilation function, and inflammatory cytokines in BA-E and BA-R patients was analyzed. The result showed that the IncRNA PVT1/miR-423-5p axis in the serum of BA-R and BA-E patients was negatively correlated with FEV /FVC, FEV1\% pred, and IL-10, and positively correlated with IgE, TNF- $a$, and IL-6 (all $P<0.01$, Table 3).

\section{Correlation of IncRNA PVT1/miR-423-5p with BA severity in $B A-E$ patients}

Furthermore, changes of IncRNA PVT1/miR-423-5p in 31 mild BA-E patients, 43 moderate BA-E patients, and 16 severe BA-E patients were analyzed and the result showed that IncRNA PVT1 and the IncRNA PVT1/miR-423-5p axis were associated with increased severity while miR-423-5p was negatively correlated with BA severity (all $P<0.01$, Figure $4 \mathrm{~A}-\mathrm{C}$ ).

\section{Discussion}

BA is a common chronic disease of childhood with increasing prevalence at an alarming rate. ${ }^{19}$ Many IncRNAs and 

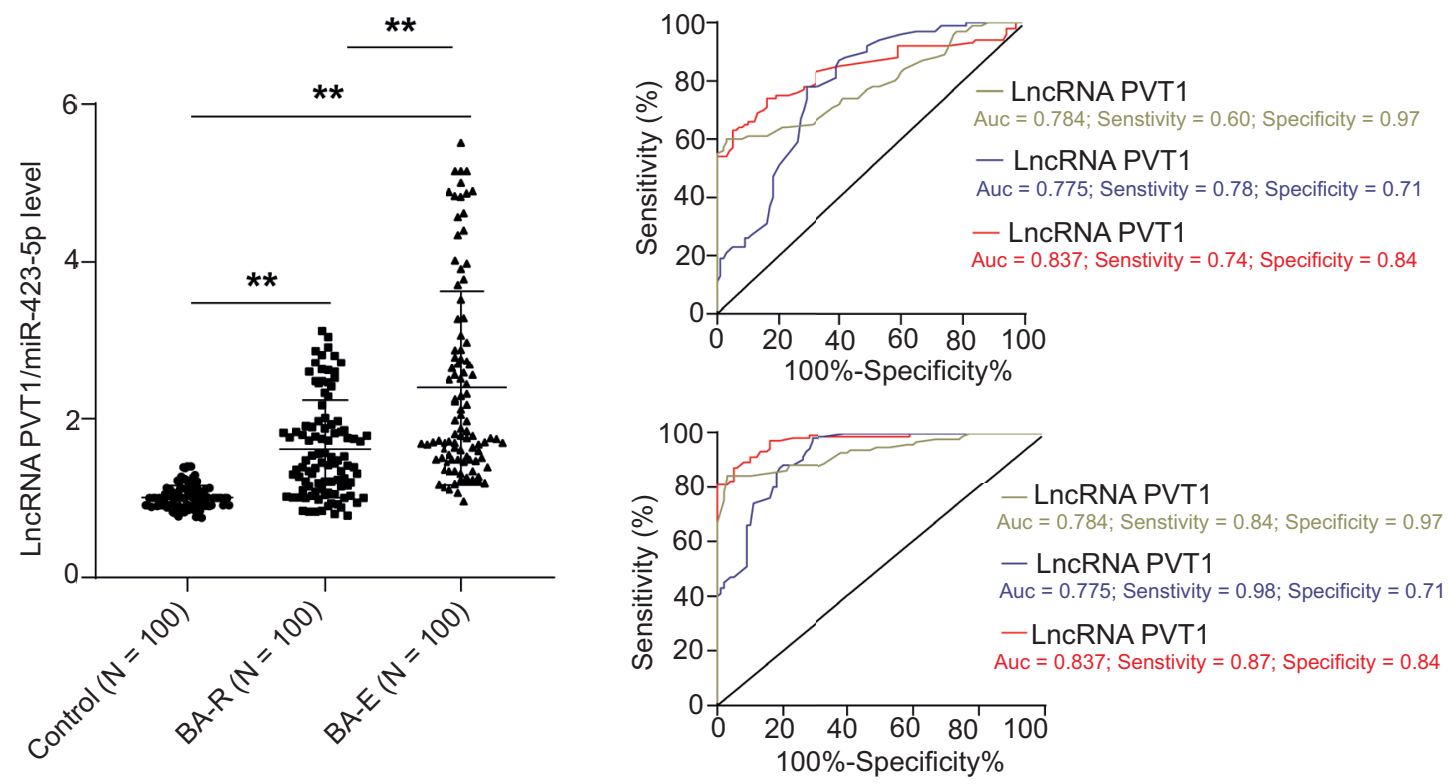

Figure 3 LncRNA PVT1/miR-423-5p axis had higher diagnostic efficiency in BA patients. (A) Changes of IncRNA PVT1/miR-423-5p expressions in BA patients; (B) Diagnostic efficiency of IncRNA PVT1/miR-423-5p axis on BA-R patients; (C) Diagnostic efficiency of IncRNA PVT1/miR-423-5p axis in BA-E patients. Data in panel C were analyzed using one-way ANOVA, followed by Tukey's multiple comparisons test. ${ }^{* *} \mathrm{P}<0.01$.

Table 3 Correlation analysis of IncRNA PVT1/miR-423-5p axis and clinical parameters.

\begin{tabular}{lcccc}
\hline Parameters & \multicolumn{4}{c}{ LncRNA PVT1/miR-423-5p } \\
\cline { 2 - 5 } & \multicolumn{2}{c}{ BA-R $(\mathrm{N}=100)$} & \multicolumn{2}{c}{ BA-E $(\mathrm{N}=100)$} \\
\cline { 2 - 5 } & $\mathrm{r}$ & $\mathrm{P}$ & $\mathrm{r}$ & $\mathrm{P}$ \\
\hline IgE $(\mathrm{IU} / \mathrm{mL})$ & 0.637 & $<0.001$ & 0.598 & $<0.001$ \\
FEV $_{1} / \mathrm{FVC}(\%)$ & -0.352 & $<0.001$ & -0.486 & $<0.001$ \\
FEV $_{1} \% \mathrm{pred}(\%)$ & -0.446 & $<0.001$ & -0.670 & $<0.001$ \\
TNF-a $(\mathrm{pg} / \mathrm{mL})^{0.371}$ & $<0.001$ & 0.566 & $<0.001$ \\
IL-6 $(\mathrm{pg} / \mathrm{mL})$ & 0.792 & $<0.001$ & 0.641 & $<0.001$ \\
IL-10 $(\mathrm{pg} / \mathrm{mL})$ & -0.469 & $<0.001$ & -0.693 & $<0.001$ \\
\hline
\end{tabular}

Note: IncRNA, long noncoding RNA; miR, microRNA; $\mathrm{BA}-\mathrm{R}$, bronchial asthma at remission; BA-E, bronchial asthma at exacerbation; IgE, Immunglobulin $\mathrm{E} \mathrm{FEV}_{1}$, forced expiratory volume in $1 \mathrm{~s}$; FVC, forced vital capacity; TNF-a, tumor necrosis factor-a; IL, interleukin.

miRNAs have been found to be potentially associated with asthma. ${ }^{20}$ This study demonstrated that IncRNA PVT1 was upregulated in the serum of BA patients while miR-423-5p was downregulated in the serum of BA patients and negatively associated with PVT1 expressions.

Generally, allergic BA is associated with the increase of serum IgE. ${ }^{21}$ In this study, the IgE level of BA-R and BA-E patients was remarkably increased and the IgE level of BA-E patients was comparatively higher than that of BA-R patients. BA severity is associated with $\mathrm{FEV}_{1} / \mathrm{FVC} .{ }^{22}$ Our study result showed that the value of $\mathrm{FEV}_{1} / \mathrm{FVC}$ and $\mathrm{FEV}_{1} \%$ pred were decreased in BA-R and BA-E patients. BA is characterized by elevated pro-inflammatory cytokines IL-6 and
TNF-a. ${ }^{23}$ Changes of IL-10 expression are also associated with $\mathrm{BA}$ incidence and severity. ${ }^{24}$ Our result showed that levels of pro-inflammatory cytokines TNF- $\alpha$ and IL- 6 were increased while anti-inflammatory cytokine IL-10 level was decreased in BA-R and BA-E patients, and the difference between $B A-R$ and $B A-E$ patients was significant. Based on a previous study, IncRNA PVT1 was clearly decreased in airway smooth muscle cells exposed to anti-asthma drugs. ${ }^{25}$ The expression level of IncRNA PVT1 was higher in the serum of children with asthma than in control children. ${ }^{26}$ In this study, expression of IncRNA PVT1 was significantly increased in the serum of BA-R and BA-E patients, and PVT1 level in BA-E patients was higher than that in BA-R patients, indicating that PVT1 expression pattern may be useful in BA diagnosis and helpful to distinguish BA-R from BA-E.

To further explore the effect of IncRNA PVT1 on BA, the downstream miRNAs of IncRNA PVT1 were investigated. miR-423-5p was found to be poorly expressed in the human airway smooth muscle cells pretreated with Th2. ${ }^{16}$ In our study, miR-423-5p expression was decreased in the serum of BA-R and BA-E patients, and miR-423-5p expression in the serum of BA-R patients was higher than that of BA-E patients. The target relation of PVT1 and miR-423-5p was verified by dual-luciferase reporter assay and expressions of IncRNA PVT1 and miR-423-5p were negatively correlated. The negative correlation of PVT1 and miR-423-5p was also found in thyroid cancer. ${ }^{27}$ Taken together, IncRNA PVT1/miR-423-5p axis might be involved in BA.

To investigate the diagnostic value of IncRNA PVT1/ miR-423-5p on BA, changes of IncRNA PVT1/miR-423-5p expressions in BA patients were analyzed. In our study, expressions of IncRNA PVT1/miR-423-5p were increased in the serum of BA-R and BA-E patients, and expressions of IncRNA PVT1/miR-423-5p in BA-E patients were higher than that in BA-R patients. The area under the ROC curve 

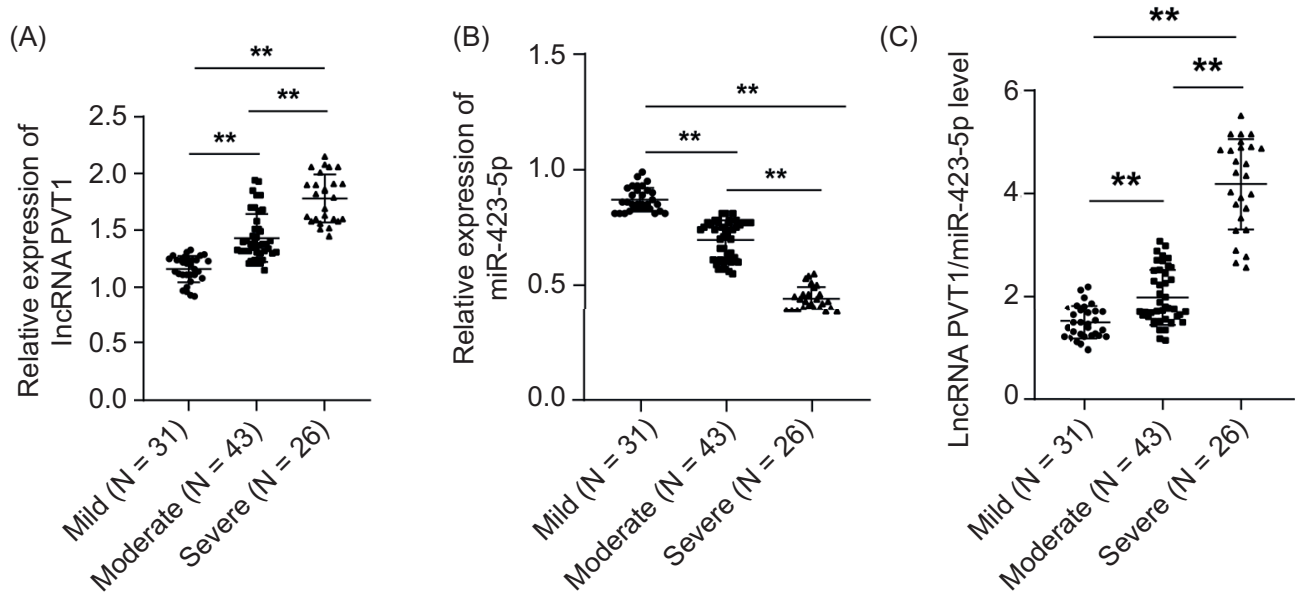

Figure 4 Correlation of IncRNA PVT1/miR-423-5p with BA severity in BA-E patients. (A) Changes of IncRNA PVT1 expression in mild, moderate, and severe BA-E patients; (B) Changes of miR-423-5p expression in mild, moderate, and severe BA-E patients; (C) Changes of IncRNA PVT1/miR-423-5p expressions in mild, moderate, and severe BA-E patients. Data were analyzed using one-way ANOVA, followed by Tukey's multiple comparisons test. ${ }^{* *} \mathrm{P}<0.01$.

of IncRNA PVT1/miR-423-5p diagnosing BA-R patients was 0.837 with sensitivity 0.74 and specificity 0.84 . The area under the ROC curve of IncRNA PVT1/miR-423-5p diagnosing BA-E patients was 0.974 with sensitivity 0.87 and specificity 0.95 . The areas under ROC curves among the three (IncRNA PVT1, miR-423-5p, and IncRNA PVT1/miR-423-5p axis) on BA-R presented no significant difference, while the area under the ROC curve of IncRNA PVT1/miR-423-5p axis diagnosing BA-R patients were higher than that of IncRNA PVT1 and miR-423-5p, indicating a higher diagnostic efficiency of IncRNA PVT1/miR-423-5p axis in diagnosing BA patients, especially acute BA-E patients. PVT1 was promising in diagnosis of asthma, with high sensitivity $(0.844)$ and specificity (0.978). ${ }^{25}$ Specifically, our results showed that IncRNA PVT1/miR-423-5p axis in the serum of BA-R and $\mathrm{BA}-\mathrm{E}$ patients was negatively correlated with $\mathrm{FEV}_{1} / \mathrm{FVC}$, FEV1\% pred, and IL-10, and positively correlated with IgE, TNF- $a$, and IL-6. The changes of IncRNA PVT1/miR-423-5p expressions in mild, moderate, and severe patients varied. Mice received ovalbumin + ozone showed higher PVT1 expression, and were associated with limited Th1/Th2like cytokines. ${ }^{25}$ PVT1 knockdown inhibited the levels of inflammatory factors in asthma. ${ }^{11}$ Altogether, IncRNA PVT1 and IncRNA PVT1/miR-423-5p axis were associated with increased severity of BA while miR-423-5p was negatively correlated with BA severity.

To summarize, this study elicited that the IncRNA PVT1/miR-423-5p axis has higher diagnostic efficiency in BA patients, especially in patients with acute BA-E, and thus provided a new reference for BA diagnosis and severity evaluation. Several limitations exist in the current study. The number of cases and events analyzed in this study was small. The sample size needs to be expanded and multi-center researches are required to further clarify the diagnostic capability of the IncRNA PVT1/miR-423-5p axis. Multi-center prospective studies should be carried out in the future to increase the reliability of the results. In the current study, we focused on the diagnostic value of the
IncRNA PVT1/miR-423-5p axis on BA patients, without considering the differences between allergic asthma and nonallergic asthma. Therefore, whether asthma patients had allergic asthma (whether antigen-specific IgE was positive) was not considered during enrollment. In future studies, we will further explore the clinical value of the IncRNA PVT1/miR-423-5p axis in patients with allergic and nonallergic asthma. Moreover, future studies will be carried out to explore the molecular regulatory mechanisms of IncRNA PVT1/miR-423-5p axis in BA.

\section{Acknowledgements}

Not applicable.

\section{Funding}

No funding was received.

\section{Competing Interests}

The authors declare that they have no competing interests.

\section{Ethics Approval}

This study was authorized by the Ethics Committee of Zibo hospital for the prevention and treatment of occupational diseases. All participants signed the informed consent before the recruitment.

\section{Consent to Participate}

Not applicable. 


\section{Availability of Data and Materials}

All the data generated or analyzed during this study are included in this published article.

\section{Authors' Contributions}

JQ was the guarantor of the integrity of the whole research, and was also responsible for the research concept, research design, definition of knowledge content, and literature research. $\mathrm{JL}$ and $\mathrm{JQ}$ were concerned with clinical research; JQ and PG were involved in experimental research; KZ and NZ were responsible for data collection; RFW and BLZ were in charge of data analysis; QJ and XSS were responsible for statistical analysis; QJ was involved in manuscript preparation; QJ and DFC were concerned with manuscript editing; DFC reviewed the manuscript. All authors read and approved the final manuscript.

\section{References}

1. Huo Y, Zhang HY. Genetic mechanisms of asthma and the implications for drug repositioning. Genes (Basel). 2018 May 3;9(5). https://doi.org/10.3390/genes9050237

2. Lambrecht BN, Hammad H, Fahy JV. The cytokines of asthma. Immunity. 2019 Apr 16;50(4):975-991. https://doi. org/10.1016/j.immuni.2019.03.018

3. Michalik M, Wojcik-Pszczola K, Paw, Wnuk M, Koczurkiewicz $D$, Sanak $P$, et al. Fibroblast-to-myofibroblast transition in bronchial asthma. Cell Mol Life Sci. 2018 Nov;75(21):39433961. https://doi.org/10.1007/s00018-018-2899-4

4. Upham JW, James LM. Remission of asthma: the next therapeutic frontier? Pharmacol Ther. 2011 Apr;130(1):38-45. https://doi.org/10.1016/j.pharmthera.2011.01.002

5. Maselli DJ, Peters Jl. Medication regimens for managing acute asthma. Respir Care. 2018 Jun;63(6):783-796. https://doi. org $/ 10.4187 /$ respcare. 05953

6. Al-Busaidi N, Habibulla Z, Bhatnagar M, Al-Lawati N, Al-Mahrouqi Y. The burden of asthma in Oman. Sultan Qaboos Univ Med J. 2015 May;15(2):e184-e190.

7. Croisant S. Epidemiology of asthma: prevalence and burden of disease. Adv Exp Med Biol. 2014;795:17-29. https://doi. org/10.1007/978-1-4614-8603-9_2

8. Narozna B, Langwinski W, Szczepankiewicz A. Non-coding RNAs in pediatric airway diseases. Genes (Basel). 2017 Nov 27;8(12). https://doi.org/10.3390/genes8120348

9. Booton R, Lindsay MA. Emerging role of microRNAs and long noncoding RNAs in respiratory disease. Chest. 2014 Jul;146(1):193-204. https://doi.org/10.1378/chest.13-2736

10. Zhang J, Zhu Y, Wang R. Long noncoding RNAs in respiratory diseases. Histol Histopathol. 2018 Aug;33(8):747-756.

11. Ma L, Zhang Q, Hao J, Wang J, Wang C. LncRNA PVT1 exacerbates the inflammation and cell-barrier injury during asthma by regulating miR-149. J Biochem Mol Toxicol. 2020 Nov;34(11):e22563. https://doi.org/10.1002/jbt.22563

12. Austin PJ, Tsitsiou C, Boardman E, Jones SW, Lindsay MA, Adcock IM, et al. Transcriptional profiling identifies the long noncoding RNA plasmacytoma variant translocation (PVT1) as a novel regulator of the asthmatic phenotype in human airway smooth muscle. J Allergy Clin Immunol. 2017 Mar;139(3):780789. https://doi.org/10.1016/j.jaci.2016.06.014

13. Li S, Ye X, Lu Y. Long non-coding RNA NEAT1 overexpression associates with increased exacerbation risk, severity, and inflammation, as well as decreased lung function through the interaction with microRNA-124 in asthma. J Clin Lab Anal. 2020 Jan;34(1):e23023. https://doi.org/10.1002/jcla.23023

14. Qiu YU, Y Wu, MJ Lin, T Bian, YL Xiao, C Qin. LncRNAMEG3 functions as a competing endogenous RNA to regulate Treg/Th17 balance in patients with asthma by targeting microRNA-17/RORgammat. Biomed Pharmacother. 2019 Mar;111:386-394. https://doi.org/10.1016/j.biopha.2018.12.080

15. Ye S, Zhu S, Feng L. LncRNA ANRIL/miR-125a axis exhibits potential as a biomarker for disease exacerbation, severity, and inflammation in bronchial asthma. J Clin Lab Anal. 2020 Mar;34(3):e23092. https://doi.org/10.1002/jcla.23092

16. Herbert C, Sebesfi M, Zeng QX, Oliver BG, Foster PS, Kumar rK. Using multiple online databases to help identify microRNAs regulating the airway epithelial cell response to a virus-like stimulus. Respirology. 2015 Nov;20(8):1206-1212. https://doi. org/10.1111/resp.12606

17. Schmittgen TD, Livak KJ. Analyzing real-time PCR data by the comparative C(T) method. Nat Protoc. 2008;3(6):1101-1108. https://doi.org/10.1038/nprot.2008.73

18. Zhang XY, Tang XY, Li N, Zhao LM, Guo Y, Li XS, et al. GAS5 promotes airway smooth muscle cell proliferation in asthma via controlling miR-10a/BDNF signaling pathway. Life Sci. 2018 Nov 1;212:93-101. https://doi.org/10.1016/j.lfs.2018.09.002

19. Toskala E, Kennedy DW. Asthma risk factors. Int Forum Allergy Rhinol. 2015 Sep;5(Suppl 1):S11-S16. https://doi.org/10.1002/ alr. 21557

20. Li Y, Yin Z, Fan J, Zhang S, Yang W. The roles of exosomal miRNAs and IncRNAs in lung diseases. Signal Transduct Target Ther. 2019;4:47. https://doi.org/10.1038/s41392-019-0080-7

21. Del Giacco S, Bakirtas R, Bel A, Custovic E, Diamant A, Hamelmann E, et al. Allergy in severe asthma. Allergy. 2017 Feb;72(2):207-220. https://doi.org/10.1111/all.13072

22. Chae EJ, Kim TB, Cho YS, Park CS, Seo JB, Kim N, et al. Airway measurement for airway remodeling defined by post-bronchodilator FEV1/FVC in asthma: investigation using inspiration-expiration computed tomography. Allergy Asthma Immunol Res. 2011 Apr;3(2):111-117. https://doi.org/10.4168/ aair.2011.3.2.111

23. Bazan-Socha S, Mastalerz L, Cybulska A, Zareba L, Kremers R, Zabczyk M, et al. Prothrombotic state in asthma is related to increased levels of inflammatory cytokines, IL-6 and TNFalpha, in peripheral blood. Inflammation. 2017 Aug;40(4):1225-1235. https://doi.org/10.1007/s10753-017-0565-x

24. Branchett WJ, Stolting H, Oliver RA, Walker SA, Puttur F, Gregory LG, et al. A T cell-myeloid IL-10 axis regulates pathogenic IFN-gamma-dependent immunity in a mouse model of type 2-low asthma. J Allergy Clin Immunol. 2020 Feb;145(2):666-678, e669. https://doi.org/10.1016/j. jaci.2019.08.006

25. Wei, Han, Dai, Guo, Zhang, Zhao, et al., Exposure to ozone impacted Th1/Th2 imbalance of $\mathrm{CD}(4+)$ T cells and apoptosis of ASMCs underlying asthmatic progression by activating IncRNA PVT1-miR-15a-5p/miR-29c-3p signaling. Aging (Albany NY). 2020 Nov 20;12(24):25229-25255. https://doi. org/10.18632/aging.104124

26. Wang Y, Luo B, Zhang W, Zhu S, Chen C, Zhou L. The IncRNA PVT1/miR-590-5p/FSTL1 axis modulates the proliferation and migration of airway smooth muscle cells in asthma. Autoimmunity. 2021 May;54(3):138-147. https://doi.org/10.108 0/08916934.2021.1897977

27. Lin QI, Qi QL, Hou S, Chen Z, Zhang L, Zhao HG, et al. LncRNA PVT1 acts as a tumor promoter in thyroid cancer and promotes tumor progression by mediating miR-423-5p-PAK3. Cancer Manag Res. 2020;12:13403-13413. https://doi.org/10.2147/ CMAR.S283443 